\title{
Lexicalidade biomédica e sua mensuração em um corpus sobre COVID-19 em língua portuguesa
}

\author{
Karhyne S. Padilha de Assis ${ }^{1}$, Camila das Merces Silva ${ }^{1}$, Janaína da Silva Leite ${ }^{\mathbf{1}}$, \\ Wellington Araujo Nogueira' ${ }^{1}$, Kenji Nose Filho', André K. Takahata ${ }^{1}$, Margarethe \\ Steinberger-Elias ${ }^{1}$.
}

${ }^{1}$ Centro de Engenharia, Modelagem e Ciências Sociais Aplicadas

Universidade Federal do ABC (UFABC) - Santo André - SP - Brasil

\{karhyne.assis, camila.merces, janaina.leite, wellington.araujo,

kenjinose, andre.t, margarethe.elias\} dufabc.edu.br

\begin{abstract}
We analyzed the biomedical lexicon of a corpus of Portuguese texts on Covid-19 extracted from the Pubmed database with the help of classic measures like lexical density and lexical diversity. Preliminary results could not show the lexical distribution in different texts genres and clinical specialties present in the corpus. Based on the concept of "biomedical lexicality", a new indicator, LexBioMed, was proposed and tested demonstrating good performance.
\end{abstract}

Resumo. Analisamos o léxico biomédico de um corpus de textos em língua portuguesa da base Pubmed sobre a Covid-19. A adoção inicial de medidas clássicas de densidade e diversidade lexical não foi capaz de evidenciar a distribuição lexical nos diferentes gêneros e especialidades clínicas de que se compõe o corpus. Com base no conceito de "lexicalidade biomédica", foi proposto e testado um novo indicador, o Lex-BioMed, com bons resultados.

\section{Introdução e motivação}

A COVID-19, inicialmente identificada em Wuhan na China em dezembro de 2019, teve aumento de casos em diversos países e continentes, até ser decretada pandêmica pela Organização Mundial da Saúde (OMS) em 11 de março de 2020 [Cucinotta e Vanelli, 2020]. A partir desta data, textos biomédicos publicados na base Pubmed foram filtrados por Leite et al. (2020) para a criação de um corpus em português a respeito da COVID-19. Com o objetivo de prover dados para uma pesquisa dos processos de simplificação da linguagem da Saúde, o corpus COVID-19 visa atender a demanda informacional de um público brasileiro leigo sobre a pandemia. Compõe-se de 254 textos do período inicial da pandemia, compreendido entre março e setembro de 2020 . O corpus é heterogêneo, distribuído entre 23 gêneros textuais e 16 especialidades clínicas. Sendo o nível lexical o de complexidade mais visível no corpus, optou-se no presente trabalho pela análise do léxico biomédico e procedeuse à contagem de types e tokens e à mensuração da diversidade ou riqueza lexical (DiL) dada pela razão entre número de types e tokens (TTR, type-token ratio) e à densidade lexical (DeL) dada pela razão entre o número de palavras de conteúdo semântico (nomes, adjetivos e verbos) e o número total de palavras do corpus [Santos et al., 2018].

O nosso problema inicial de pesquisa foi como identificar automaticamente termos biomédicos de difícil compreensão e convertê-los em expressões acessíveis. Tomou-se como ponto de partida a hipótese de que os índices de densidade e de diversidade lexical seriam capazes de apontar os gêneros de maior complexidade, isto é, onde haveria maior concentração de types. Métodos clássicos como Flesch-Kincaid, partições morfológicas e outros foram temporariamente deixados de lado, colocando-se o texto especializado como 
foco da pesquisa. "Tendo-se o texto como foco, deixa de fazer sentido que se continue estudando somente os termos, de forma que se passa a englobar os modos de dizer peculiares de cada área de conhecimento" [Finatto, 2004a, p. 348].

Linguagens de especialidade, como é o caso da biomédica, tem um comportamento diferenciado nos estudos de corpora: "(...) a partir da observação da linguagem especializada em corpora que se percebe mais francamente como a observação de termos é somente um pequeno passo na observação do texto especializado" [Perna, Delgado e Finatto, 2010 p.138]. Estudo de Zilio (2009) sobre textos científicos de Cardiologia e Radiologia compara a distribuição lexical entre as duas especialidades e atribui as convergências a fatores textuais: “(...) se não houvesse no corpus de Radiologia um artigo que se ocupasse do coração, somente dois dos compostos estudados seriam comuns aos dois corpora" (p.142).

A observação inicial sobre o comportamento lexical das linguagens de especialidades nos textos do corpus e a indefinição das medidas de densidade lexical nesse contexto levou a busca de um novo indicador da lexicalidade no corpus. Propomos aqui o conceito de "lexicalidade biomédica" ou "densidade lexical biomédica" para identificar com maior segurança o espaço lexical que é das especialidades biomédicas e diferenciá-lo de um léxico fronteiriço revelado em gêneros menos técnicos. O problema de pesquisa foi revisto, tornando-se imperativo reconhecer no corpus um repertório de termos biomédicos, de modo a identificar sua distribuição no corpus sobre COVID-19. Investigamos o novo indicador de lexicalidade para verificar se seria capaz de cumprir uma função distribucional que as simples densidade e diversidade lexicais não lograram alcançar.

\section{Materiais e Métodos}

Como já descrito na Seção 1, seguindo [Leite et al., 2020], foi obtido um corpus de textos em língua portuguesa a respeito da COVID-19, a partir de textos indexados na base científica Pubmed do período entre março e setembro de 2020, totalizando 254 textos. Os textos foram categorizados manualmente conforme gêneros, utilizando informações fornecidas pela base, e conforme especialidades clínicas, de acordo com o nome da revista, título do artigo ou palavras-chave do texto. As distribuições dos textos nas classes obtidas se encontram nas Tabelas 2 e 3. Após a fase preliminar de filtragem, limpeza e compilação, buscou-se nomear os arquivos já em formato .txt, tokenizar e fazer a anotação morfossintática das classes de palavras (PoS, parts of speech) com o uso do analisador e corretor gramatical CoGrOO [Silva, 2013]. O resultado para as classes de palavras de conteúdo semântico (nome, verbo e adjetivo) se encontra na Tabela 1.

Com finalidade de caracterizar e descrever o léxico biomédico do corpus, foram calculados os índices da DeL e da DiL. A DeL descreve a proporção de palavras de conteúdo pelo número total de palavras em cada texto e de acordo com [Ure, 1971] [Johansson, 2008], o valor resultante desse indicador expressa a concentração de conteúdo lexical presente em um determinado texto. Um texto com alta densidade lexical contém mais palavras de conteúdo, enquanto um texto com baixa densidade lexical é composto por palavras funcionais (preposições, conjunções, artigos, pronomes, verbos modais e auxiliares). Já a DiL pode ser descrita pela TTR. Entretanto, a TTR tende a possuir valor menor em textos com maior número de tokens ou a possuir valor maior em textos com menor número de tokens, fazendo com que o seu uso torne enviesada a comparação entre dois textos ou corpora com número de tokens diferentes [Johansson, 2008]. Para mitigar esse efeito, utilizamos também o vocabulário teórico (VT) ou theoretical vocabulary [Broeder, Extra \& van Hout 1986], em que a proporção de types é calculada para subconjuntos de tamanho fixo com $N$ tokens, no nosso caso $N=100$, amostrados aleatoriamente. Em nosso trabalho, 
mostramos resultados obtidos a partir da realização de $S=100$ amostragens aleatórias distintas para cada grupo de texto.

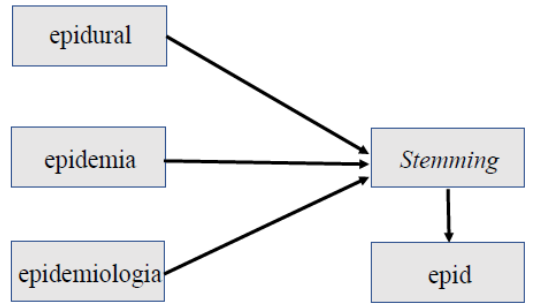

Figura 1: Stemming

Tabela 1: Resultados para as PoS por subcorpora

\begin{tabular}{|c|c|c|c|c|c|c|c|c|c|}
\hline Especialidade & $\begin{array}{c}\mathrm{N}^{\circ} \mathrm{de} \\
\text { publicações }\end{array}$ & $\begin{array}{c}\text { Total de } \\
\text { tokens }\end{array}$ & $\mathrm{n}$ & $\operatorname{adj}$ & $\mathrm{v}$ & n-adj & $\mathrm{n}(\%)$ & $\operatorname{adj}(\%)$ & $\mathrm{v}(\%)$ \\
\hline Saúde Pública & 55 & 83028 & 40997 & 11376 & 17707 & 117 & 49,38 & 15,03 & 21,33 \\
\hline Epidemiologia & 36 & 50810 & 24542 & 7381 & 10267 & 88 & 48,30 & 14,53 & 20,21 \\
\hline Cardiologia & 30 & 37891 & 16429 & 6690 & 7981 & 65 & 46,00 & 17,66 & 21,06 \\
\hline Enfermagem & 24 & 46410 & 23265 & 6476 & 10083 & 68 & 50,13 & 13,09 & 21,73 \\
\hline Cirurgia & 17 & 25098 & 10693 & 3548 & 5138 & 22 & 42,60 & 14,14 & 20,47 \\
\hline Outros & 16 & 21446 & 11656 & 3827 & 5498 & 49 & 54,35 & 17,84 & 25,64 \\
\hline Nefrologia & 14 & 14930 & 6882 & 2196 & 3122 & 21 & 46,10 & 14,71 & 20,91 \\
\hline Terapia Intensiva & 11 & 15534 & 7424 & 2209 & 3219 & 20 & 47,79 & 14,22 & 20,72 \\
\hline Pneumologia & 10 & 4791 & 2289 & 823 & 1000 & 3 & 47,78 & 17,18 & 20,87 \\
\hline Relatório Imagens & 9 & 3717 & 1939 & 755 & 757 & 3 & 46,79 & 20,31 & 20,37 \\
\hline Não Classificados & 8 & 6413 & 3062 & 876 & 1368 & 10 & 47,75 & 13,66 & 21,33 \\
\hline Anestesiologia & 7 & 6672 & 3073 & 1071 & 1520 & 4 & 46,06 & 16,05 & 22,78 \\
\hline Clínica Geral & 5 & 2733 & 1280 & 399 & 624 & 8 & 46,83 & 14,60 & 22,83 \\
\hline Fonoaudiologia & 5 & 4131 & 2094 & 604 & 941 & 3 & 50,69 & 14,62 & 22,78 \\
\hline Pediatria & 4 & 5679 & 2569 & 919 & 1158 & 7 & 45,24 & 17,94 & 20,39 \\
\hline Saúde Primária & 3 & 2177 & 1192 & 330 & 386 & 3 & 50,16 & 15,16 & 17,73 \\
\hline
\end{tabular}

Para a identificação das palavras de domínio biomédico em toda extensão do corpus foi usado, como primeiro passo, o stemming, sendo empregado para implementação o RSLP Stemmer [Orengo e Huyck 2001] que tem como objetivo remover os sufixos, para reduzir o número de palavras, conforme ilustrado na Figura 1. Observamos que originalmente o corpus possui 31.123 types. Após o stemming esse número se reduziu para 11.513 raízes distintas. Após a obtenção das raízes, foi criada uma lista de pares do tipo (raiz, type), em que o segundo elemento consiste em um type escolhido ao acaso dentre as palavras do corpus que podem ser formadas com o uso da respectiva raiz. Assim, para a raiz "epid" na Figura 1, o par formado poderia ser ("epid", “epidural"). Os types de todos os 11.513 pares foram analisados por três pesquisadores de processamento de linguagem natural (PLN) em textos biomédicos, sendo o time formado por duas mulheres e um homem, em que dois são docentes universitários (com formação em engenharia elétrica e linguística) e uma é estudante de mestrado (com formação em análise e desenvolvimento de sistemas), com idades entre 34 e 69 anos. Procedeu-se à análise de modo a comparar a identificação de palavras com uso prevalente no domínio biomédico, resultando em 2.258 raízes associadas a palavras no domínio biomédico.

A partir dessa identificação de raízes biomédicas, passamos para a etapa de análise de cada subcorpus formado por textos agrupados por gênero ou especialidade com cálculo do DeL, DiL-TTR e DiL-VT. Constatando que tais índices não foram capazes de mostrar a distribuição lexical por gênero e por especialidade, criamos um novo indicador que pudesse medir a lexicalidade biomédica (Lex-BioMed), que consiste na proporção do número de types que possuem raízes biomédicas em conjuntos de tamanho fixo (no nosso caso $N=100$ ) 
obtidos aleatoriamente no grupo de textos em análise. Assim como para a DiL-VT, mostramos resultados para Lex-BioMed obtidos a partir da realização de $S=100$ amostragens aleatórias distintas.

\section{Resultados}

Nas Tabelas 2 e 3 são apresentados os resultados para os subcorpora formados a partir da categorização dos textos por especialidades clínicas e por gênero textual, respectivamente. Ao analisarmos o DiL-TTR, observamos que o principal fator de influência para essa métrica foi o número de tokens. A influência dos números de tokens na medida não se observou com o DeL e com o DiL-VT, mas, como mostrado nas Tabelas 2 e 3, os valores obtidos revelaramse similares entre os subcorpora analisados, não permitindo uma discriminação clara a respeito do conteúdo biomédico presente nos respectivos subconjuntos de textos. Contrastando com as métricas mais tradicionais, ao analisarmos a Lex-BioMed, é possível observar que se torna possível diferenciar subcorpora ou grupos de subcorpora diferentes a partir dessa métrica, o que também é corroborado pela Figura 2. Por exemplo, para especialidades, as categorias com maior Lex-BioMed foram "Cardiologia" e "Relatório de Imagens" com 25,29\% e 24,57\% respectivamente, enquanto as categorias de menor LexBioMed foram "Saúde Pública" e "Saúde Primária", com, respectivamente, 9,17\% e 8,54\%. Na categorização por gêneros, os de maior Lex-BioMed foram "Aprendendo por Imagens" e "Imagens Pneumologia" com proporção de 29,84\% e 27,35\% respectivamente. Na Figura 2, os box-plot indicam que a distribuição da proporção de palavras com raízes biomédicas em conjuntos de $N=100$ palavras escolhidas aleatoriamente é diferente entre as categorias, principalmente dentre as com maior e menor valor de Lex-BioMed. Ao ordenar as subcategorias em ordem decrescente de Lex-BioMed, como na Tabela 2 e na Figura 2(a), observamos que podemos agrupar as especialidades em dois subconjuntos. O primeiro composto por "Cardiologia", "Relatório Imagens", "Nefrologia", "Pediatria", "Anestesiologia", "Cirurgia", "Fonoaudiologia", "Pneumologia" e "Terapia Intensiva"; e o segundo formado por "Outros", "Epidemiologia", "Clínica Geral", "Enfermagem", "Não Classificados", "Saúde Pública" e "Saúde Primária". Nota-se que, em linhas gerais, no primeiro grupo encontram-se especialidades médicas mais específicas, como Cardiologia, Radiologia e Nefrologia, enquanto, no segundo grupo, encontram-se categorias indefinidas, como "Outros" e "Não Classificados", categorias não médicas, como "Enfermagem" e "Saúde Pública", ou de caráter mais amplo, como "Clínica Geral".

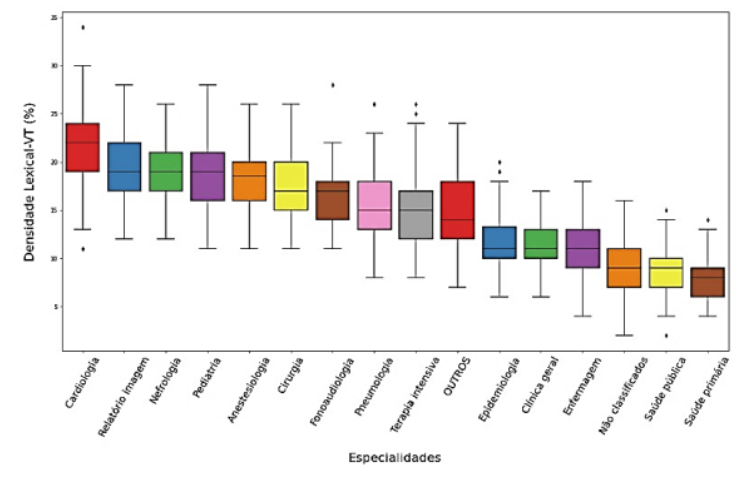

(a)

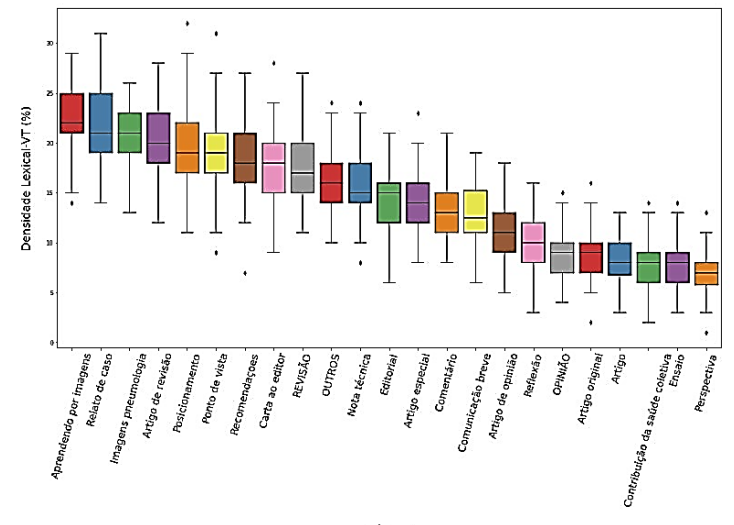

(b)

Figura 2: Box-plot descrevendo a distribuição da proporção de raízes biomédicas em $S=100$ amostras aleatórias obtidas para cálculo de Lex-BioMed para cada (a) especialidade e (b) gênero textual. 
Tabela 2. Resultados das características por especialidade

\begin{tabular}{l|r|r|r|r|r|r|r|r|r}
\hline \multicolumn{1}{c|}{ Especialidade } & $\begin{array}{c}\mathrm{N}^{\circ} \\
\text { publicacões }\end{array}$ & $\begin{array}{c}\text { Total de } \\
\text { tokens }\end{array}$ & $\begin{array}{c}\text { Total de } \\
\text { types }\end{array}$ & $\begin{array}{c}\text { Tokens } \\
\text { biomédicos }\end{array}$ & $\begin{array}{c}\text { Types } \\
\text { biomédicos }\end{array}$ & DeL (\%) & DiL-TTR (\%) & DiL-VT (\%) & Lex-BioMed (\%) \\
\hline Cardiologia & 30 & 37891 & 8063 & 9311 & 912 & 43,24 & 21,28 & 76,28 & 25,29 \\
Relatório Imagens & 9 & 3717 & 1414 & 940 & 186 & 40,89 & 38,04 & 76,24 & 24,57 \\
Nefrologia & 14 & 14930 & 4568 & 3409 & 461 & 42,11 & 30,60 & 75,09 & 22,83 \\
Pediatria & 4 & 5679 & 2696 & 1200 & 390 & 39,67 & 47,47 & 76,31 & 22,54 \\
Anestesiologia & 7 & 6672 & 2765 & 1504 & 319 & 40,68 & 41,44 & 74,83 & 21,13 \\
Cirurgia & 17 & 25098 & 6320 & 5177 & 554 & 42,75 & 25,18 & 75,58 & 20,63 \\
Fonoaudiologia & 5 & 4131 & 1975 & 753 & 203 & 40,64 & 47,81 & 74,27 & 19,29 \\
Pneumologia & 10 & 4791 & 2168 & 924 & 208 & 40,94 & 45,25 & 74,95 & 18,23 \\
Terapia Intensiva & 11 & 15534 & 4147 & 2563 & 420 & 41,53 & 26,70 & 75,32 & 16,50 \\
Outros & 16 & 21446 & 6103 & 3485 & 448 & 42,41 & 28,46 & 75,95 & 16,25 \\
Epidemiologia & 36 & 50810 & 8813 & 6605 & 448 & 44,50 & 17,35 & 74,24 & 13,00 \\
Clínica Geral & 5 & 2733 & 1495 & 327 & 100 & 40,35 & 54,70 & 76,11 & 11,96 \\
Enfermagem & 24 & 46410 & 9033 & 5479 & 416 & 42,92 & 19,46 & 75,84 & 11,81 \\
Não Classificados & 8 & 6413 & 2597 & 660 & 132 & 40,78 & 40,50 & 75,64 & 10,29 \\
Saúde Pública & 55 & 83028 & 14091 & 7611 & 534 & 48,02 & 16,97 & 76,20 & 9,17 \\
Saúde Primária & 3 & 2177 & 1176 & 186 & 61 & 39,03 & 54,02 & 74,30 & 8,54 \\
\hline
\end{tabular}

Tabela 3. Resultados das características por gênero

\begin{tabular}{l|r|r|r|r|r|r|r|r|r}
\hline \multicolumn{1}{|c|}{ Gênero } & $\begin{array}{r}\text { Total } \\
\text { publicacões }\end{array}$ & $\begin{array}{c}\text { Total de } \\
\text { tokens }\end{array}$ & $\begin{array}{c}\text { Total de } \\
\text { types }\end{array}$ & $\begin{array}{c}\text { Tokens } \\
\text { biomédicos }\end{array}$ & $\begin{array}{c}\text { Types } \\
\text { biomédicos }\end{array}$ & DeL (\%) & DiL-TTR (\%) & DiL-VT (\%) & Lex- BioMed (\%) \\
\hline Aprendendo por Imagem & 3 & 868 & 484 & 259 & 98 & 45,28 & 55,76 & 74,20 & 29,84 \\
Imagens Pneumologia & 35 & 21521 & 6846 & 3652 & 514 & 43,90 & 31,81 & 74,79 & 27,35 \\
Relato de Caso & 3 & 8898 & 3820 & 972 & 179 & 41,88 & 42,93 & 76,55 & 24,85 \\
Artigo de Revisão & 14 & 33093 & 7350 & 8121 & 849 & 41,80 & 22,21 & 74,88 & 24,54 \\
Recomendações & 17 & 17876 & 5440 & 1955 & 249 & 42,93 & 30,43 & 74,65 \\
Posicionamento & 24 & 51996 & 9887 & 5716 & 347 & 42,71 & 19,01 & 75,29 & 22,82 \\
Ponto de Vista & 6 & 7594 & 3185 & 911 & 168 & 43,29 & 41,94 & 76,18 & 22,32 \\
Cartas ao Editor & 8 & 7607 & 2773 & 1684 & 325 & 41,38 & 36,45 & 75,99 & 22,14 \\
Revisão & 4 & 4437 & 2139 & 419 & 80 & 41,60 & 48,21 & 76,12 & 21,13 \\
Nota Técnica & 3 & 1888 & 992 & 309 & 89 & 44,16 & 52,54 & 75,79 & 20,16 \\
Outros & 6 & 5027 & 2195 & 751 & 124 & 48,52 & 43,66 & 75,50 & 19,29 \\
Artigo Especial & 11 & 11081 & 3727 & 2529 & 387 & 39,90 & 33,63 & 75,77 & 18,11 \\
Editorial & 20 & 23653 & 6410 & 4284 & 658 & 42,67 & 27,10 & 76,58 & 17,26 \\
Comentário & 28 & 11612 & 4330 & 2454 & 448 & 42,89 & 37,29 & 75,30 & 16,97 \\
Comunicação Breve & 6 & 13652 & 4125 & 2752 & 459 & 38,49 & 30,22 & 73,91 & 16,37 \\
Artigo de Opinião & 6 & 6854 & 2873 & 1703 & 466 & 41,39 & 41,92 & 73,46 & 15,95 \\
Reflexão & 26 & 51975 & 9074 & 5683 & 354 & 41,90 & 17,46 & 73,63 & 14,94 \\
Opinião & 8 & 8556 & 3037 & 1365 & 208 & 40,51 & 35,50 & 75,80 & 14,87 \\
Artigo & 3 & 479 & 329 & 131 & 62 & 41,85 & 68,68 & 74,11 & 12,00 \\
Contribuições da Saúde Coletiva & 8 & 9855 & 3497 & 1901 & 322 & 43,44 & 35,48 & 74,00 & 10,99 \\
Artigo Original & 3 & 6755 & 2630 & 1508 & 360 & 40,84 & 38,93 & 74,88 \\
Ensaio & 9 & 1690 & 4936 & 2917 & 449 & 43,17 & 29,21 & 75,44 \\
Perspectiva & 3 & 4863 & 2132 & 723 & 120 & 41.77 & 43.84 & 75.99 & 10,94 \\
& & & & & & 10,93 \\
& & & & &
\end{tabular}

A partir dos resultados da Tabela 3 e da Figura 2(b), pode-se observar também que o léxico de diferentes gêneros pode ser diferenciado com uso da Lex-BioMed, em especial gêneros como "Aprendendo por Imagem" e "Perspectiva". Na Figura 3 é mostrada a distribuição conjunta entre os gêneros e as especialidades clínicas, onde as linhas e as colunas estão ordenadas por ordem decrescente de Lex-BioMed. Pode-se observar que, grosso modo, gêneros com maior valor de Lex-BioMed são constituídos de textos de especialidades que também possuem maior valor do índice, como "Aprendendo por Imagem", constituído em sua totalidade por textos da especialidade "Relatório Imagens" e "Relato de Caso", constituído majoritariamente por textos de "Cardiologia", ambas as especialidades com alta Lex-BioMed. Mesmo gêneros que sugerem textos mais opinativos, como "Posicionamento" e "Ponto de Vista", possuem relativa alta Lex-BioMed, por serem constituídos exclusivamente por textos de Cardiologia. Além disso, gêneros com baixa Lex-BioMed estão, em geral, associados às especialidades com o mesmo comportamento. Por exemplo, gêneros como "Artigo Original" e "Artigo" possuem maior prevalência de especialidades como "Epidemiologia", "Enfermagem" e "Saúde Pública". Os gêneros que possuem os menores valores de Lex-BioMed são "Ensaio" e "Perspectivas", com textos que descrevem o contexto da pandemia, ao invés de assuntos técnicos do ponto de vista clínico. É o caso 
dos textos "COVID-19, as fake news e o sono da razão comunicativa gerando monstros: a narrativa dos riscos e os riscos das narrativas" [Vasconcellos-Silva e Castiel, 2020] e "Da Tuberculose ao COVID-19: Legitimidade Jurídico-Constitucional do Isolamento/Tratamento Compulsivo por Doenças Contagiosas em Portugal" [Peixoto et al., 2020]. Os gêneros "Ensaio" e "Perspectivas" possuem maior prevalência de especialidades associadas à baixa Lex-BioMed. A Figura 3 ainda indica uma justificativa para a "Pediatria", especialidade mais geral, possuir valor maior de Lex-BioMed, do que especialidades mais específicas, como, por exemplo, "Pneumologia". Observa-se nesses casos participação majoritária de textos de gêneros como "Relato de Caso", "Artigo de Revisão" e "Imagens Pneumologia", que possuem alta Lex-BioMed como em "Pediatria", enquanto em "Pneumologia" se observa a predominância de textos dos gêneros "Cartas ao Editor" e "Editorial”, de menor índice.

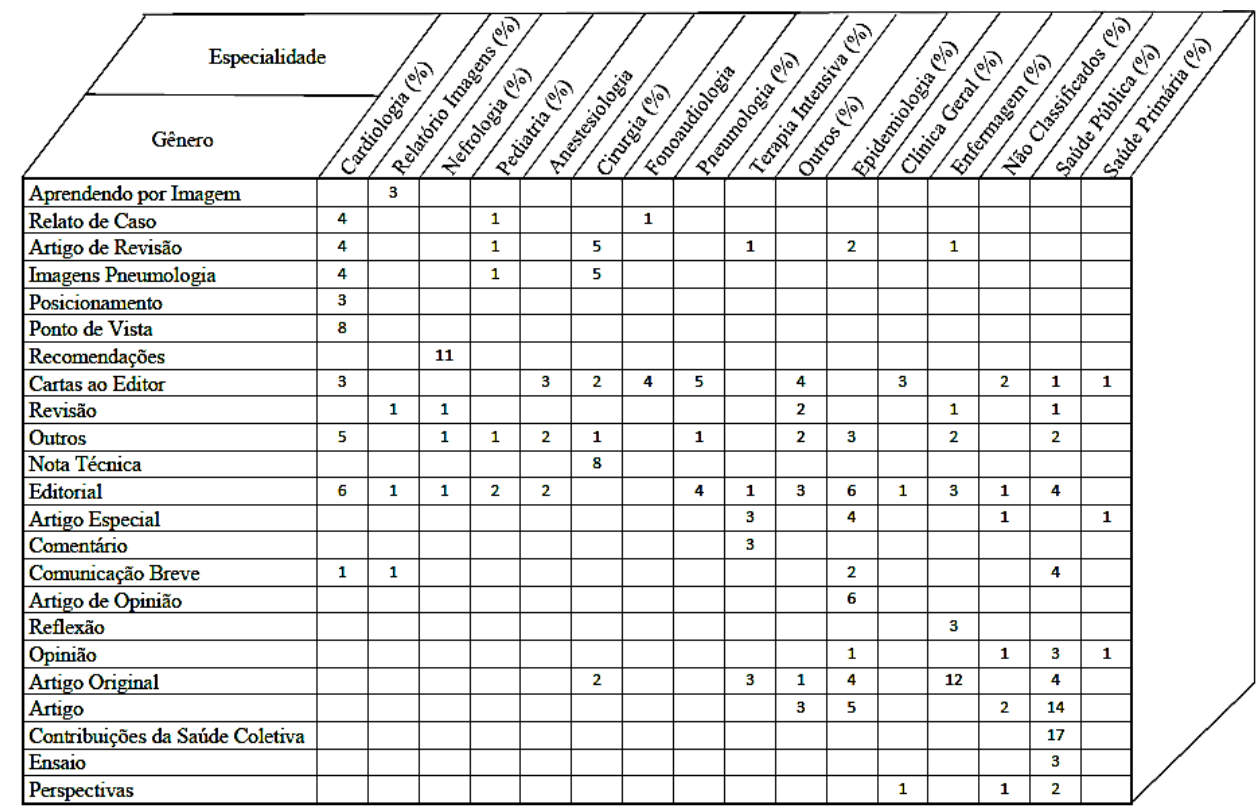

Figura 3: Distribuição conjunta dos textos do corpus por especialidades e gêneros

\section{Conclusões}

Mostramos neste trabalho que as medidas clássicas de diversidade e densidade lexical não são adequadas para mensurar o léxico de linguagens de especialidade como a biomédica. Com base no conceito de "lexicalidade biomédica", o novo indicador proposto Lex-BioMed foi capaz de revelar a distribuição lexical nos diferentes gêneros e especialidades clínicas presentes no corpus Covid-19 tomado como referência. Os resultados mostraram que os índices de lexicalidade biomédica caem em contextos fronteiriços e mais genéricos em relação a áreas mais técnicas da medicina como a Cardiologia. O recurso de vocabulário teórico utilizado também se revelou interessante para contornar o problema da variação de número de tokens entre os textos do corpus.

Dando seguimento à pesquisa, deverá ser buscada uma ampliação do corpus Covid19 ora composto de 254 textos e restrito à fase inicial de publicações sobre a doença, eventualmente acrescendo-se períodos posteriores que facultem uma análise de teor mais diacrônico. A ampliação do corpus também contempla a possibilidade de estudos comparados com corpora de outra natureza, por exemplo, construídos com base em um léxico mais popular dirigido ao público leigo. Tais resultados sugerem que outras linguagens de especialidade poderão ser investigadas em trabalhos futuros, tendo como horizonte a 
hipótese de que seu léxico também seria sensível a uma mensuração de maior aderência ao texto especializado.

Quanto à identificação manual dos termos biomédicos no corpus, cabem algumas considerações finais. Em primeiro lugar, a filtragem dos termos poderá no futuro ser melhor testada mediante consulta ampla a especialistas da área biomédica com experiência em temas associados à Covid-19. Em segundo lugar, a exploração dos termos biomédicos relacionados ao corpus da pandemia poderá ser enriquecida pelo contraponto com vocabulários eletrônicos, glossários e ontologias disponíveis sobre a Covid em português, ou mesmo aqueles em língua estrangeira que possam ser submetidos a tradução. Em terceiro lugar, a caracterização inicial do léxico da Covid baseado apenas em unigramas, tal como apresentado aqui, certamente ganhará se vier a incorporar multipalavras e collocations biomédicas. A testagem do indicador Lex-BioMed, de modo a abranger itens nocionais de formação complexa, abrirá caminho para uma abordagem da complexidade vocabular biomédica em associação a outros níveis linguísticos - sintático, semântico, pragmático e discursivo. Por fim, em quarto lugar, um estudo acurado do comportamento das PoS nas várias especialidades e gêneros de que se compõe o corpus estudado poderá refinar a distribuição lexical mostrada aqui. Para além da constatação feita aqui de que os nomes são as "âncoras nocionais" em todas as especialidades clínicas, um estudo de verbos biomédicos como "acometer" ou "diagnosticar", ou de adjetivos como "anestésico" ou "pulmonar", poderá diferenciar classes com regime mais definido ou indefinido de distribuição no corpus.

\section{Agradecimentos}

O presente trabalho foi realizado com o apoio da Coordenação de Aperfeiçoamento de Pessoal de Nível Superior - CAPES.

\section{Referências}

V. Kannan e S. Gurusamy (2014), "Preprocessing Techniques for Text Mining - An Overview", International Journal of Computer Science \& Communication Networks, V. 5, p. 7-16.

Leite, J.S., Takahata, A.K., Steinberger-Elias, M.(2020) Elaboração de corpus biomédico em Português sobre o Covid-19. Journal of Health Informatics: Número Especial CBIS Congresso Brasileiro de Informática em Saúde. Dezembro p.242-247. http://www.jhisbis.saude.ws/ojs-jhi/index.php/jhi-sbis/article/view/821

Leite, J.S., Takahata, A.K., Steinberger-Elias, M. (2020) “Criação e análise de amostras de corpora em Português Brasileiro para detecção automática de expressões complexas em textos sobre Covid-19". In: XXVII Brazilian Congress on Biomedical Engineering. Proceedings of CBEB 2020, October 26-30, Vitoria, Brazil. https://www.springer.com/gp/book/9783030706005

Orengo, V. \& Huyck, C. (2001) "A stemming algorithm for the Portuguese language". In Proceedings of the Eighth International Symposium on String Processing and

Information Retrieval (SPIRE 2001), (p. 186-193). Laguna de San Rafael, Chile: IEEE Computer Society Press.

Aluísio, S. M.; Almeida, G. M. D. B. (2006) "O que é e como se constrói um corpus? Lições aprendidas na compilação de vários corpora para pesquisa linguística Calidoscópio", São Leopoldo, V. 4, n.3. p.155-177. 
Celso Romão Cardoso De Almeida Júnior (2017). "Proposta de um Sistema Automático de Avaliação de Redações do Enem, Foco na Competência 1: Demonstrar Domínio da Modalidade Escrita Formal da Língua Portuguesa". Dissertação de Mestrado.

Cucinotta, Domenico, and Maurizio Vanelli. (2020) "WHO declares COVID-19 a pandemic". Acta Bio Médica: Atenei Parmensis 91.1 (2020): p.157.

Vasconcellos-Silva, P. R., \& Castiel, L. D. (2020) "COVID-19, “As fake news e o sono da razão comunicativa gerando monstros: a narrativa dos riscos e os riscos das narrativas". Cadernos de Saúde Pública, V. 36, n. 7, p.1-6.

Peixoto, V. R., Mexia, R., Santos, N. D. S., Carvalho, C., \& Abrantes, A. (2020) "Da tuberculose ao COVID-19: legitimidade jurídico-constitucional do isolamento/tratamento compulsivo por doenças contagiosas". In Portugal. Acta Médica Portuguesa, V. 33, p.225-228.

Krieger, M. da G, Finatto, M. J. B. (2004) “Introdução à terminologia: teoria \& prática”. São Paulo:Contexto, p.348.

Ure, J. (1971) "Lexical density and register differentiation". In: G.E. PERREN; J.L.M. TRIM (eds.), Applications of linguistics. Selected papers of the Second International Congress of Applied Linguistics. Cambridge/Londres, Cambridge University Press, p. 443-452.

Johansson, V. (2008) "Lexical diversity and lexical density in speech and writing: a developmental perspective". Lund University, Department of Linguistics and Phonetics: Working Papers, V. 53, p.61-79.

Broeder, P., Coenen, J., Extra, G., van Hout, R., \& Zerrouk, R. (1986) "Ontwikkelingen in het Nederlandstalig lexicon bij anderstalige volwassenen: Een macro- en microperspectief". In J. Creten, G. Geerts, \& K. Jaspaert (Eds.), Werk-in-uitvoering: Momentopname van de sociolinguïstiek in België en Nederland, p.39-57.

Perna, L. Cristina; Delgado, K. Heloísa; Finatto, J. Maria. (2010) "Linguagens Especializadas em CORPORA. Modos de Dizer e Interfaces de Pesquisa". EDIPUCSEditora Universitária da Pontifícia Universidade Católica do Rio Grande do Sul, Porto Alegre, p.138.

Santos, E. S. et al. (2018) "Diversidade e densidade lexical em textos escritos por alunos recém-alfabetizados: um estudo descritivo de produções individuais e em díades". Calidoscópio Revista Unisinos, V. 16, n.1, p.25-32.

Silva W.D.C.M. (2013) “Aprimorando o corretor gramatical CoGrOO”, Dissertação de Mestrado em Ciência da Computação, IME-USP, São Paulo, SP.

Zilio, L. (2009) “Colocações especializadas e Komposita: um estudo contrastivo alemãoportuguês na área de cardiologia”. Porto Alegre: UFRGS. Dissertação de Mestrado. PPGLETRAS/UFRGS. 